Rev. Adm. Saúde - Vol. 18, № 73, out. - dez. 2018

http://dx.doi.org/10.23973/ras.73.145

ARTIGO ORIGINAL

\title{
Processo de auditoria de contas em um hospital público: mapeamento das glosas técnicas em cirurgias ortopédicas
}

Audit process in a public hospital: mapping of the technical glosses in surgeries orthopedics

\section{Eduardo Neves da Cruz de Souza1, Cleyber Henrique Santos ${ }^{2}$, Priscilla Higashi $^{3}$, Isabel Fernandes de Souza ${ }^{4}$}

1. Enfermeiro. Especialista em auditoria de serviços de saúde. Professor colaborador do curso de enfermagem da Universidade Estadual do Oeste do Paraná, Foz do Iguaçu PR

2. Enfermeiro

3. Enfermeira. Doutoranda em enfermagem em saúde pública na Escola de Enfermagem de Ribeirão Preto da USP, Ribeirão Preto SP

4. Graduado em ciência da computação. Docente de informática no IFPR, Foz do Iguaçu PR

\section{RESUMO}

O presente estudo tem como objetivo mapear, por meio do levantamento dos dados de contas auditadas, as glosas ocorridas nas cirurgias ortopédicas realizadas no centro cirúrgico de um hospital público de médio porte do município de Foz do Iguaçu, Paraná. Método: Pesquisa observacional, exploratória, documental, retrospectiva de abordagem quantitativa, com levantamento dos prontuários de cirurgias ortopédicas que apresentaram glosas no faturamento, pelo período de janeiro de 2016 a dezembro de 2017. Resultados: Constatou-se que em 454 prontuários levantados surgiram 117 glosas, totalizando $\mathrm{R} \$ 67.994,38$ de perda técnicas geradas pela ortopedia. Conclusão: Deste modo, esta pesquisa disponibilizou à administração do hospital um banco de dados com informações referentes as glosas levantadas juntamente com a receita institucional gerada nestes prontuários. 
Palavras-chave: Centro Cirúrgico; Ortopedia; Custos hospitalares; Auditoria financeira; Hospital público.

\begin{abstract}
Objective: The objective of this study is to map, by means of a survey of audited accounts data, the glosses of orthopedic surgeries performed in the surgical center of a medium-sized public hospital in the municipality of Foz do Iguaçu, Paraná.

Method: Observational, exploratory, documental, retrospective study of quantitative approach, with a survey of records of orthopedic surgeries that presented glosses in the billing, for the period from January 2016 to December 2017. Results: It was found that in 454 medical records raised, 117 totaling $R$ \$ 67,994.38 in loss of techniques generated by orthopedics. Conclusion: In this way, this research made available to the hospital administration a database with information referring to the glosses raised along with the institutional revenue generated in these medical records.
\end{abstract}

Keywords: Surgical Center; Orthopedics; Hospital costs; Financial audit; Public hospital

\title{
INTRODUÇÃO
}

A inconsistência nos registros de materiais e medicamentos utilizados e os erros desses registros pela equipe no centro cirúrgico, ambos geram prejuízos financeiros à instituição. Tais inconsistências constituem-se em um dos focos de atenção dos auditores, cujo serviço integra o controle administrativo-financeiro das organizações ${ }^{(1)}$.

No centro cirúrgico, a auditoria reflete diretamente o serviço de enfermagem, pois, a partir dos cuidados prescritos e das anotações realizadas são justificados 0 pagamento de equipamentos, gases, materiais, medicamentos e diversos procedimentos realizados $(2,3,4)$.

A imprecisão e a incompletude nas anotações, acompanhado da falta de embasamento ou justificativas para realização de determinado procedimento, estão entre os principais motivos das perdas técnicas envolvendo a equipe de enfermagem ${ }^{(5,6)}$ e equipe médica ${ }^{(7)}$.

Assim, ao não preencher completamente os prontuários ou inserir inconsistências, ambas atividades estão diretamente relacionadas ao número de glosas da instituição. As glosas são sinônimos de prejuízo no faturamento organizacional (8).

Torna-se importante destacar que o prontuário médico, principal ferramenta do auditor e da equipe assistencial, contém um conjunto de procedimentos realizados 
podendo compor uma série de laudos. Esses por sua vez, vão desde o momento da abertura da Autorização de Internação Hospitalar $(\mathrm{AlH})$ até seu respectivo fechamento. Ao ser inspecionado, cada procedimento pode ser aprovado ou reprovado pela auditoria externa, constituído assim glosas parciais ao faturamento. Deste modo, um prontuário pode conter mais de uma glosa registrada ${ }^{(7,9,10,11)}$.

Ao evitar glosas, a conta pública do paciente é impactada, evitando as perdas e cancelamentos desnecessários, especialmente nos setores mais críticos, como o centro cirúrgico ${ }^{(8,12,13)}$.

Neste sentido, o presente estudo objetivou mapear, por meio do levantamento dos dados de contas auditadas, as glosas ocorridas nas cirurgias ortopédicas realizadas no centro cirúrgico de um hospital público de porte médio, e seus desdobramentos sobre os resultados financeiros da instituição, no período compreendido entre janeiro de 2016 a dezembro de 2017.

\section{METODOLOGIA}

Trata-se de um estudo observacional, exploratório, documental, retrospectivo de abordagem quantitativa, realizado no setor de centro cirúrgico de um hospital público de médio porte do município de Foz do Iguaçu (PR).

Os dados foram coletados por meio de levantamento nos prontuários do centro cirúrgico, exclusivamente das cirurgias ortopédicas que apresentaram glosas no faturamento.

Para coleta de dados foram utilizados o Serviço de Arquivamento Médico e Estatística (SAME), concomitante com o sistema informatizado de prontuário eletrônico TASY (software de gestão hospitalar da empresa Philips adotada pela instituição pesquisada). Assim, ambos possibilitaram o acesso aos prontuários de janeiro de 2016 a dezembro de 2017 - período de investigação da pesquisa. Cada prontuário foi averiguado manualmente, de modo aleatório, mapeando: itens glosados (materiais e procedimento médico); valor glosado; valor da conta e categoria profissional responsável pela glosa.

A partir da confirmação da glosa, se obteve as justificativas registradas pelo auditor, tais como: a) procedimento não compatível com o sistema de gerenciamento da tabela de procedimentos, medicamentos e órtese, prótese e materiais especiais (SIGTAP) do Sistema Único de Saúde (SUS); b) sem exames de raios X; c) sem descrição de procedimento; d) código na classificação internacional de doença (CID) não compatível.

Os prontuários levantados representam estatisticamente uma amostra, calculada com índice de confiabilidade de $95 \%$ e margem de erro de 5\%.

Para o tratamento dos dados foi utilizado o software Excel 2010 para a estatística descritiva, em que foram calculados os valores da média, mediana, desvio padrão. A apresentação dos dados foi feita no formato gráfico em tabelas. 
O projeto de pesquisa foi submetido ao Comitê de Ética em Pesquisa da Universidade Estadual do Oeste do Paraná (UNIOESTE), sendo aprovado no parecer consubstanciado de número 2.515.363.

\section{RESULTADOS}

No total foram levantados aleatoriamente 454 prontuários, de um montante de 2.818 submetidos à ortopedia, referente aos anos de 2016 a 2017. Esse levantamento documental demandou 61 horas e 50 minutos de pesquisa e avaliação de dados.

Considerando a amostra levantada - 454 (100\%) prontuários, 117 (25,7\%) demostraram glosas no faturamento. Nesse conjunto, a partir dos 117 (100\%) prontuários glosados, foram encontrados pacientes submetidos aos procedimentos de cirurgia ortopédica, na faixa etária de 0 a 89 anos, com média de idade de 40 (DP 19). Quanto ao gênero, 81 (69,2\%) eram do sexo masculino e $36(30,8 \%)$ correspondia ao sexo feminino.

Ao considerar os 117 prontuários, obteve-se um valor total faturado, pelo Hospital Campo de Estudo (HCE), de $\mathrm{R} \$ 110.784,88$. Nesses prontuários, foram encontradas 204 glosas, superior ao número de prontuários levantados, totalizando $\mathrm{R} \$ 67.994,38$ de perda técnicas.

De acordo com a Tabela 1, mínimo e máximo contido nas receitas hospitalares foram de $R \$ 115,45$ a $R \$ 4.114,81$ com mediana de $R \$ 569,34$.

Houve variação do valor glosado entre $R \$ 11,84$ e $R \$ 1.602,18$, com média de $R \$$ 393,03 (DP R $\$ 270,58$ ). Assim, se observa que a porcentagem do valor glosado, a partir do valor total de perda, variou de $0,1 \%$ a $2,3 \%$ e a quantidade média de itens glosados foi de 1,18 (DP 0,62), variando de 1 a 6 itens por conta hospitalar/prontuário.

Tabela 1. Distribuição dos prontuários glosados $(n=117)$ relativo ao valor da receita, valor glosado, porcentagem do valor glosado e quantidade de itens glosados, de janeiro de 2016 a dezembro de 2017.

\begin{tabular}{|c|c|c|c|c|c|c|c|}
\hline Variáveis & $\begin{array}{r}\text { Valor } \\
\text { Total (R\$) }\end{array}$ & $\begin{array}{r}\text { Média } \\
(\mathrm{R} \$)\end{array}$ & $\begin{array}{r}\text { Desvio } \\
\text { Padrão } \\
(R \$)\end{array}$ & $\begin{array}{l}\text { Mín. } \\
\text { (R\$) }\end{array}$ & $\begin{array}{r}\text { Máx. } \\
\text { (R\$) }\end{array}$ & $\begin{array}{r}\text { Mediana } \\
(\mathrm{R} \$)\end{array}$ & $\mathbf{n}$ \\
\hline Valor da receita & $110.784,88$ & 893,43 & 861,06 & 115,45 & $4.114,81$ & 563,38 & 117 \\
\hline Valor glosado & $67.994,38$ & 393,03 & 270,58 & 11,84 & $1.602,18$ & 382,18 & 117 \\
\hline Valor glosado (\%) & 100 & 0,6 & 0,4 & 0,1 & 2,3 & 0,5 & 117 \\
\hline Quantidade itens & 204 & 1,18 & 0,62 & 1 & 6 & 1 & 117 \\
\hline
\end{tabular}


No Gráfico 1, o desvio padrão $(\mathrm{R} \$ 893,43)$ expressivo gerou valores dispersos da média. Assim, podemos observar o valor máximo, arrecadado pela instituição, de $\mathrm{R} \$$ $4.114,81$ com maior frequência de procedimentos com custo de $R \$ 338,03$ a $R \$$ $1.130,41$.

Assim, com o mesmo mecanismo de interpretação dos dados, verifica-se que o valor total glosado chegou a $R \$ 1.602,18$ sendo a maior concentração na faixa de $R \$$ 151,66 a $\mathrm{R} \$ 543,08$.

Gráfico 1. Demonstração da volatilidade financeira gerada pelas glosas e pelas receitas dos prontuários glosados $(n=117)$, de janeiro de 2016 a dezembro de 2017.

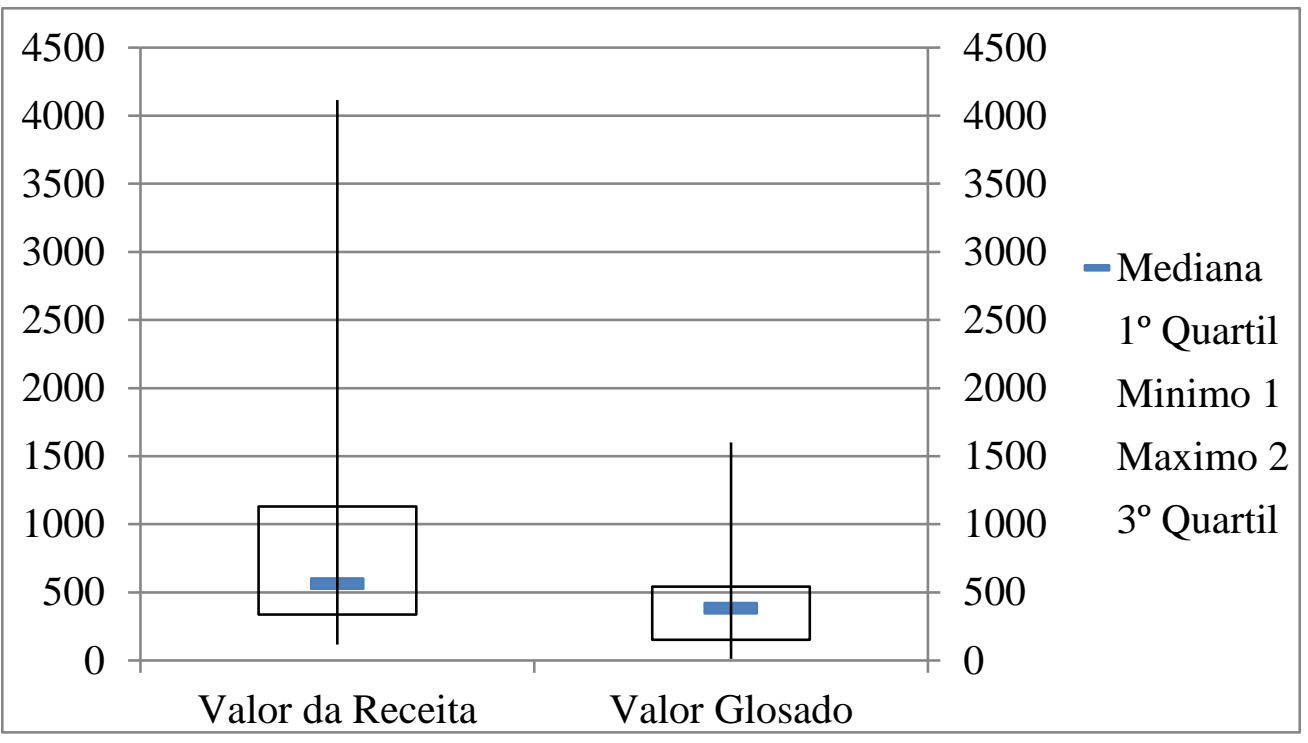

Na Tabela 2, a partir das 204 (100\%) glosas, observou-se que 132 $(75,7 \%)$ corresponderam a procedimentos médicos, gerando uma média de $\mathrm{R} \$$ 429,40 (DP $R \$ 228,65$ ), com variação de $R \$ 11,84$ a $R \$ 1.602,18$. Destes procedimentos, 72 itens $(24,3 \%)$ corresponderam a materiais ortopédicos que gerou de $R \$ 15,34$ a $R \$ 1.513,63$ de valor glosado com mediana $R \$ 121,18$. 
Tabela 2. Distribuição dos prontuários glosados relativa ao valor total glosado, valor glosado por procedimento e valor glosado por materiais, de 2016 a dezembro de 2017.

\begin{tabular}{|c|c|c|c|c|c|c|c|}
\hline Variáveis & $\begin{array}{r}\text { Valor Total } \\
(\mathbf{R} \$)\end{array}$ & $\begin{array}{r}\text { Média } \\
(\mathbf{R} \$)\end{array}$ & $\begin{array}{r}\text { Desvio } \\
\text { Padrão } \\
\text { (R\$) }\end{array}$ & $\begin{array}{l}\text { Mín. } \\
\text { (R\$) }\end{array}$ & Máx. (R\$) & $\begin{array}{r}\text { Mediana } \\
(\mathbf{R} \$)\end{array}$ & $\mathbf{n}$ \\
\hline Valor total glosado & $67.994,38$ & 393,03 & 270,58 & 11,84 & $1.602,18$ & 382,18 & 204 \\
\hline $\begin{array}{l}\text { Valor glosado } \\
\text { procedimento }\end{array}$ & $56.251,71$ & 429,40 & 228,65 & 11,84 & $1.602,18$ & 473,83 & 132 \\
\hline $\begin{array}{l}\text { Valor glosado } \\
\text { materiais }\end{array}$ & $11.742,67$ & 279,59 & 352,08 & 15,34 & $1.513,63$ & 121,18 & 72 \\
\hline
\end{tabular}

Demonstra-se no Gráfico 2, que o valor máximo glosado nos procedimentos chegou a $R \$ 1.602,18$, sendo o maior volume entre $R \$ 246,97$ a $R \$ 543,08$. Da mesma forma, verifica-se que o valor máximo dos materiais glosados chegou a $R \$ 1.513,63$ sendo a maior concentração na faixa de $R \$ 55,42$ a $R \$ 361,81$.

No que se refere aos prontuários glosados, no ano de 2016 foram identificados 43 prontuários, encontrando 37 de glosas relacionadas a procedimentos e 35 relacionadas a materiais. Assim, totalizaram 72 glosas, gerando um valor de prejuízo de $\mathrm{R} \$ 21.022,19$.

No ano de 2017, foram levantados 74 prontuários, apresentando 95 de glosas relacionadas aos procedimentos e 37 de perdas relacionadas aos materiais. Deste modo, totalizaram 132 glosas, gerando um total de $R \$ 46.972,19$ de valor glosado, ou seja, perda para a instituição.

Gráfico 1. Demonstração da volatilidade financeira gerada pelos procedimentos e materiais dos prontuários glosados, de janeiro de 2016 a dezembro de 2017. 


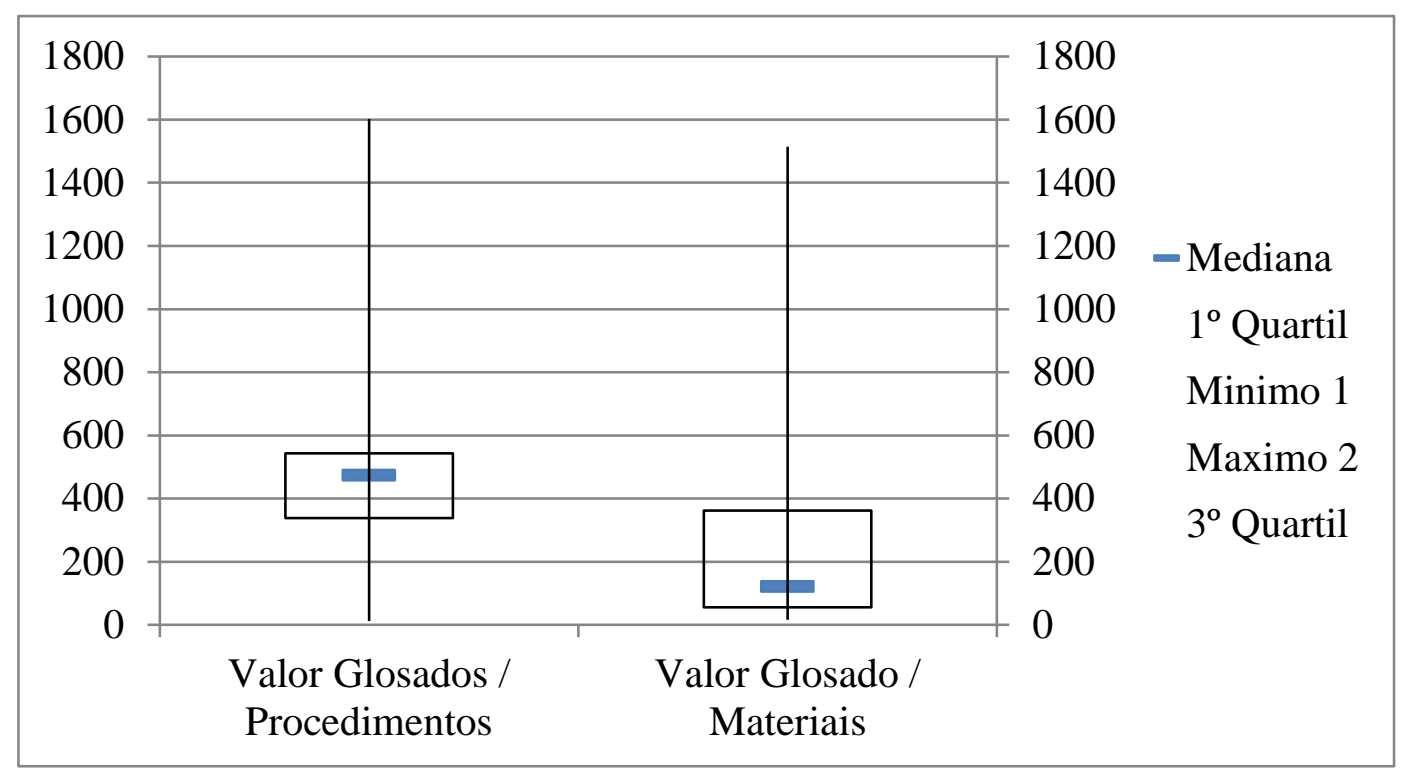

As glosas foram identificadas, e classificadas, na presente pesquisa em: a) procedimento não compatível / Tabela SIGTAP; b) sem descrição da glosa / auditor; c) via acesso d) sem raios X; e) procedimento não descrito / cirurgião; f) não identificação o motivo da glosa / pesquisador; g) sem raios $X$ e sem descrição da glosa / auditor; h) CID não compatível. Assim, apresenta-se na Tabela 3 as descrições das 204 glosas.

Esse quantitativo foi identificado em 173 descrições, representadas por: 68 procedimentos não compatíveis com a Tabela SIGTAP (39\%); 53 sem descrição da glosa pelo médico auditor (30,6\%); 23 procedimentos que já estavam inclusos no procedimento cirúrgico geral (Via Acesso) (13,3\%); 14 glosas sem raios $X(8,1 \%) ; 6$ procedimentos não descritos pelo médico cirurgião (3,5\%); 4 que não foram identificados o motivo da glosa pelo pesquisador (2,3\%); 3 sem descrição da glosa pelo médico auditor e sem exames de raios $X(1,7 \%)$; e 2 CIDs não compatíveis $(1,2 \%)$.

Tabela 3. Distribuição das descrições das glosas, das quantidades apresentadas e de seus respectivos valores relativos à amostra dos 117 prontuários do período de janeiro de 2016 a dezembro de 2017.

\begin{tabular}{lrrrr}
\hline $\begin{array}{l}\text { Descrição da } \\
\text { Glosa }\end{array}$ & $\begin{array}{r}\text { Quantidade } \\
\text { de } \\
\text { Descrições }\end{array}$ & $\%$ & $\begin{array}{r}\text { Valor Glosado } \\
\text { (R\$) }\end{array}$ & $\begin{array}{r}\text { Valor da Receita } \\
\text { (R\$) }\end{array}$ \\
\hline $\begin{array}{l}\text { Procedimento não } \\
\text { compatível / Tabela }\end{array}$ & 68 & $39,3 \%$ & 26148,11 & 51124,29 \\
SIGTAP & & & & \\
\end{tabular}




\begin{tabular}{|c|c|c|c|c|}
\hline $\begin{array}{l}\text { Sem descrição da } \\
\text { glosa / auditor }\end{array}$ & 53 & $30,6 \%$ & 19497,69 & 20775,4 \\
\hline Via acesso & 23 & $13,3 \%$ & 11317,6 & 14527,03 \\
\hline Sem raios $X$ & 14 & $8,1 \%$ & 4563,42 & 12449,629 \\
\hline $\begin{array}{l}\text { Procedimento não } \\
\text { descrito / cirurgião }\end{array}$ & 6 & $3,5 \%$ & 2812,83 & 5628,78 \\
\hline $\begin{array}{l}\text { Não identificado o } \\
\text { motivo / } \\
\text { pesquisador }\end{array}$ & 4 & $2,3 \%$ & 1590,83 & 1643,43 \\
\hline $\begin{array}{l}\text { Sem descrição da } \\
\text { glosa / auditor e } \\
\text { sem raios X }\end{array}$ & 3 & $1,7 \%$ & 1138,64 & 3907,74 \\
\hline CID não compatível & 2 & $1,2 \%$ & 925,26 & 728,58 \\
\hline Total Geral & 173 & $100 \%$ & $\mathrm{R} \$$ 67.994,38 & $\mathrm{R} \$ 110.784,879$ \\
\hline
\end{tabular}

Ao levantar os dados e considerando as 2818 cirurgias ortopédicas realizadas no ano de 2016 e 2017, estimam-se pela amostra mapeada 454 prontuários. Do total de prontuário, 117 foram glosados. Assim, o prejuízo total foi de $R \$$ $67.994,38$. Ao tomarmos como base esse quantitativo para uma estimativa bianual, o HCE deixou de receber um montante de $\mathrm{R} \$ 424.964,87$.

Torna-se importante ressaltar que o HCE não possui um sistema de análise prévia das contas hospitalares, deixando assim, de fazer ajustes positivos (inclusões) e negativos (exclusões) o qual justifica o recebimento dos procedimentos e evita glosas ${ }^{(1)}$.

\section{DISCUSSÃO}

A amostra mapeada no estudo revelou que $25,7 \%(n=454)$ apresentaram glosas parciais no faturamento. Resultado semelhante ao encontrado no estudo de Zunta (2016) ${ }^{(10)}$, no qual as glosas ocorreram em 18\% $(n=384)$ da amostra de prontuários. A pesquisa da autora foi realizada em instituição particular e contou com uma equipe de auditoria de enfermagem que utilizava recurso de glosas.

Para que as instituições hospitalares mantenham seu equilíbrio financeiro, são necessários investimentos contínuos em ações educativas direcionadas a melhorias dos registros hospitalares ${ }^{(10)}$. 
Quando há anotações inconsistentes, ilegíveis, subjetivas ou até mesmo incompatibilidade de anotações geram desperdícios no faturamento da instituição ${ }^{(5,9,14)}$. Neste sentido, observou-se no estudo de Foz do Iguaçu que as principais distorções que acarretaram nas glosas nos prontuários da ortopedia não foram apenas relacionadas aos registros inconsistentes, incompatíveis ou com letras ilegíveis. Os resultados levantados revelaram 7 temas que acarretaram na geração das glosas ou na não identificação desta, sendo todos estes expostos e discutidos a seguir.

\section{Procedimento não compatível / Tabela SIGTAP}

Evidenciou-se, assim, que no HCE a principal fonte de desperdício e de geração de glosas nas cirurgias ortopédicas, está relacionada aos procedimentos não compatíveis com a Tabela SIGTAP.

Em estudo realizado por Camargo (2017) (15), em um hospital público de ensino, foram mapeadas 4.539 cirurgias que utilizaram órtese, prótese e materiais especiais (OPM), apontou que $3 \%(n=143)$ procedimentos realizados não estavam inclusos na Tabela SIGTAP, se caracterizando por não compatibilidade com os procedimentos ofertados pelo SUS.

Outro fator no estudo de Foz do Iguaçu é o não uso ou consulta à Tabela SIGTAP.

De acordo com a última atualização da Tabela SIGTAP, em 2008, encontra-se cadastrados no sistema 533 procedimentos de recursos médicos implantáveis, sendo 309 de órteses, próteses e materiais especiais relativos aos processos cirúrgicos ${ }^{(16)}$. Assim, essa lista poderia ter sido utilizada, previamente, nos procedimentos registrados nos documentos que foram mapeados no estudo do HCE.

A título de exemplificar a importância da consulta à Tabela SIGTAP, estudos apresentaram que é indispensável que no decorrer do processo de auditoria implantes de OPME, deve-se analisar também o tipo de procedimento cirúrgico realizado se averiguando se está ou não compatível com SIGTAP e quais são suas principais indicações ${ }^{(13,17)}$. As OPME são representativas na ortopedia, tanto que podem alcançar até $87 \%$ do custo total do procedimento ${ }^{(18)}$.

Para Camargo (2017) ${ }^{(15)}$, a diminuição da ocorrência das glosas, relacionadas à incompatibilidade com a Tabela SIGTAP, se tornará possíveis a partir de novas pesquisas que analisem os dispositivos que constam nesta tabela. Tais como: as compatibilidades existentes, os valores cobrados e as quantidades autorizadas para os procedimentos.

\section{Sem descrição da glosa / auditor}

Na pesquisa do HCE, encontrou-se, no estudo, que devido à falta da descrição da glosa pelo auditor não foi possível identificar o motivo da perda. 
Segundo o Manual de Auditoria SUS (2015) ${ }^{(19)}$, as comprovações da auditoria, juntamente com suas respectivas avaliações, opiniões, recomendações e conclusões devem ser informadas por meio de um relatório técnico. Assim, para que o auditor possa desempenhar sua missão, com efetividade, é essencial estar a par dos princípios éticos. Assim, o auditor deve adquirir técnicas e procedimentos reconhecidos e aprovados para obter e organizar evidências, formar convicção, sobre as circunstâncias, e emitir um parecer tecnicamente fundamentado.

Ao envolver o SUS, é importante ter como diretriz o uso dos recursos públicos, que são obtidos através do pagamento de tributos pela sociedade. Assim, toda movimentação de verba pública deve observar os princípios da moralidade, publicidade e da eficiência $(20,21,22)$.

\section{Via de acesso}

No que se refere às glosas dos procedimentos realizados que não estavam inclusos na cirurgia principal, foi caracterizado pelo auditor como "via de acesso". Nos manuais de auditoria do SUS não foi possível encontrar tal descrição, no entanto, no sistema de saúde suplementar brasileiro, o Manual de Classificação Brasileira Hierarquizada de Procedimentos Médicos (2016) (23:212-27) pontua que:

[...] Quando previamente planejada, ou quando se verificar, durante $o$ ato cirúrgico, a indicação de atuar em vários órgãos ou regiões ou em múltiplas estruturas articulares a partir da mesma via de acesso, a quantificação do porte da cirurgia será a que corresponder, por aquela via, ao procedimento de maior porte, acrescido de $50 \%$ do previsto para cada um dos demais atos médicos praticados, desde que não haja um código específico para o conjunto (23:212-27).

Sendo assim, ao se tratar da saúde suplementar, percebe-se que há dispositivos legais que fundamenta e padroniza este tipo de cobrança, o que não foi encontrado no presente estudo.

\section{Sem raios $X$}

Nos casos de implante, o Manual Técnico Operacional do Sistema de Informação Hospitalar, aprovado pela Portaria MS/GM no 396/2000, prevê a obrigatoriedade de anexar no prontuário exames de controle radiológicos (filmes e laudos) pré e pós-operatório com identificação do paciente ${ }^{(9)}$.

Cabe ressaltar, que além dos raios $\mathrm{X}$ anexo ao prontuário do paciente, é obrigatório anexar também a Nota Fiscal do produto implantável (9).

\section{Procedimento não descrito / cirurgião}


Com relação aos procedimentos não descritos pelo cirurgião, Guerrer et al (2014) (24) afirma que não se pode eximir os profissionais de saúde da responsabilidade ética, legal, jurídica e comunicativa da documentação e anotação das ações prestadas ou das justificativas por elas não terem sido realizadas.

Pesquisas complementam ${ }^{(5,14)}$ que devido à falta de registro torna-se, mais difícil o acompanhamento da evolução da patologia do paciente. Em muitos casos, ocasionando danos à assistência prestada e à comunicação da equipe multiprofissional, uma vez que o prontuário é o principal meio de comunicação entre a equipe.

Para Rosa (2017) ${ }^{(7)}$, os profissionais que estão envolvidos nos cuidados devem anotar, no prontuário, todos os procedimentos realizados, de forma clara, detalhada e legível, pois este é um documento legal.

No que tange o profissional enfermeiro, segundo a Resolução do Conselho Federal de Enfermagem (COFEN) ํo 429/2012, é responsabilidade e dever dos profissionais de enfermagem registrar no prontuário do paciente, as informações inerentes ao processo de cuidar e ao gerenciamento de processos de trabalho. Ambos necessários para assegurar a continuidade e a qualidade da assistência ${ }^{(25)}$.

\section{Não identificado o motivo / pesquisador}

No que se refere ao auditor, um dos motivos que levaram a não identificação de algumas descrições, foram relacionadas ao não entendimento da letra registrada juntamente com a não familiarização das siglas por parte do pesquisador.

De acordo com o Manual de Auditoria do SUS (2015), as análises realizadas pelos auditores devem estar bem documentadas, a ponto de poderem ser reproduzidas ${ }^{(19)}$.

\section{CID não compatível}

Quanto aos procedimentos que apresentaram CID não compatível, dados similares foram encontrados no Manual de Orientações Técnicas Sobre Auditoria na Assistência Ambulatorial e Hospitalar (2016). Tal descrição está relacionada com uma das frequentes acometidas pelo profissional de saúde ${ }^{(9)}$.

\section{CONSIDERAÇÕES ADICIONAIS DOS PESQUISADORES À PESQUISA}

Conforme exposto e considerando que o HCE obteve uma perda financeira $\mathrm{R} \$$ $67.994,38$ somente nos 117 prontuários levantados, com intuito de evitar estas 
perdas que comprometem a receita institucional, muitos hospitais públicos e privados aderem a um sistema de pré-análise de contas hospitalares ${ }^{(1,10)}$.

Pesquisa realizada pela equipe de auditores do Instituto do Coração do Hospital das Clínicas da Faculdade de Medicina da Universidade de São Paulo analisou 2.613 prontuários por meio do sistema de pré-análise de contas hospitalares as glosas ocorridas no mês de janeiro a dezembro de 2011 . Constatou-se que se não fosse o sistema de pré-análise de contas hospitalares a instituição teria uma perda financeira estimada em $\mathrm{R} \$ 628.554,55$ no referido ano ${ }^{(1)}$.

Assim, caso este trabalho de pré-análise de contas hospitalares fosse realizado no HCE, de acordo com estudos, a instituição teria menos perdas, pois os profissionais teriam a oportunidade de verificar, reparar e prevenir seus erros, extraindo daí uma efetividade maior dos processos de formação de conta $(1,10,24)$.

\section{CONCLUSÃO}

Neste estudo, verificou-se um alto índice de glosas na amostra analisada - 454 prontuários - ficando acima de 25\%, com 117 prontuários glosados e 204 glosas levantadas. Deste modo, esta pesquisa disponibilizou à administração do hospital um banco de dados de informações referente as glosas levantadas.

Cabe ressaltar que apesar das buscas continuas a literatura não se encontrou estudos envolvendo glosas na ortopedia em hospitais públicos, indicando a necessidade do desenvolvimento de estudos que permitem o aprofundamento desta temática.

\section{REFERÊNCIAS}

1. GUERRER, Gabriela Favaro Faria; LIMA, Antônio Fernandes Costa; CASTILHO, Valéria. Estudo da auditoria de contas em um hospital de ensino. Revista Brasileira de Enfermagem, v. 68, n. 3, p. 414-420, 2015.

2. DE OLIVEIRA, Driely Reis; JACINTO, Silvia Maria; SIQUEIRA, Cibele Leite. Auditoria de enfermagem em Centro Cirúrgico. Normas para

publicação/Publishingguide, v. 14, p. 151, 2013.

3. DE SOUZA, Maíra Pereira; CERETTA, Luciane Bisognin; SORATTO, Maria Tereza.

Auditoria Concorrente no Centro Cirúrgico: Concepções dos Enfermeiros.

Saúde e Pesquisa, v. 9, n. 2, p. 263-272, 2016. 
4. GROSSI, Luciane Mandia; PISA, Ivan Torres; MARIN, Heimar de Fátima. Oncoaudit: development and evaluation of an application for nurse auditors. Acta Paulista de Enfermagem, v. 27, n. 2, p. 179-185, 2014.

5. DE SOUZA, Mariana Silva Mendes; DE OLIVEIRA FIORAVANTI, Silvia Gabriela; COLAVOLPE, Verena Costa. Registro de enfermagem: desafio para as instituições hospitalares na redução de glosas. 2016.

6. PADILHA, Elaine Fátima; Fernandez Lourenço HADDAD, Maria do Carmo; Misue MATSUDA, Laura. Qualidade dos registros de enfermagem em terapia intensiva: avaliação por meio da auditoria retrospectiva. Cogitare Enfermagem, v. 19, n. 2, 2014.

7. ROSA, Marisa Rodrigues da. Padronização da gestão do processo de auditoria interna em um hospital privado na cidade de Santa Maria. São Leopoldo: UNISINOS, 2017. 55 f. 2017. Dissertação (Mestrado em Enfermagem) - Programa de Pós-Graduação em Enfermagem, Universidade do Vale do Rio dos Sinos, São Leopoldo, RS, 2017.

8. DA SILVA, Karla Rona; DE OLIVEIRA LIMA, Marina Dayrell; DE SOUSA, Marco Aurélio. Auditoria: ferramenta de enfermagem para melhoria da qualidade assistencial. Revista Eletrônica Gestão \& Saúde, v. 7, n. 2, p. 793-810, 2016.

9. BRASIL. Secretaria de Gestão Estratégica e Participativa Departamento Nacional de Auditoria do SUS. Auditoria nas Assistências Ambulatorial e Hospitalar no SUS: orientações técnicas. 1a edição. Brasília - DF, 2016.

10. ZUNTA, Raquel Silva Bicalho. 0 gerenciamento de custos relativos às glosas técnicas de um centro cirúrgico: um estudo de caso. São Paulo: USP, 2016. 119 f. Tese (Doutorado em Enfermagem) - Programa de PósGraduação em Enfermagem na Saúde do Adulto, Escola de Enfermagem da Universidade de São Paulo, São Paulo, 2016.

11. BRASIL. Ministério da Saúde. Portaria № 113, de setembro de 1997: considera os preceitos constitucionais que asseguram o acesso universal e igualitário às ações e serviços para promoção, proteção e recuperação da saúde. Disponível em:

http://bvsms.saude.gov.br/bvs/saudelegis/sas/1997/prt0113 0409 1997.ht $\underline{\mathrm{ml}}$

12. VIEIRA, Ana Paula Timoteo. Enfermeira auditora uma ferramenta importante para a qualidade do serviço em saúde em hospitais privados. Revista Especialize On-line IPOG - Goiânia - 8ª Edição no 009 Vol.01/2014 dezembro/2014.

13. DE SOUZA, Maria Gerusa Silva; DE SOUZA, Carla Giselly; CINTRA, Grace Mary Gomes. Glosas de Materiais Especiais de um Hospital Privado em Recife-PE. Revista de Psicologia, v. 10, n. 30, p. 357-366, 2016. 
14. CLAUDINO, Hellen Gomes; GOUVEIA, Eloise Maria de Lima; SANTOS, Sérgio Ribeiro dos Santos; LOPES, Maria Emília Limeira. Auditoria em registros de enfermagem: revisão integrativa da literatura. Rev. Enferm. UERJ, v. 21, n. 3, p. 397-402, 2013.

15. CAMARGO, Telma Aparecida de. Custos de órteses, próteses e materiais especiais não contemplados no Sistema Unico de Saúde (SUS) em hospital de ensino brasileiro. São Paulo, Botucatu 2017. Dissertação (Mestrado em Enfermagem) - Programa de Pós-Graduação em Enfermagem, da Universidade Estadual Paulista - UNESP, Faculdade de Medicina de Botucatu, 2017.

16. MALAVOLTA EA, ASSUNÇÃO JH, BERALDO RA, PINTO GMR, GRACITELLI MC, FERREIRA NETO A. Reparo do manguito rotador no Sistema Único de Saúde: tendência brasileira de 2003 a 2015. Rev Bras Ortop. v 52, n. 4, p.501-505, nov. 2017.

17. MACHADO, Gustavo Silveira. Órteses e próteses no Sistema Único de Saúde. Consultoria Legislativa. Câmara dos Deputados, abril de 2018.

18. BEREDJIKLIAN, Pedro K. Prescrição de Preços para Implantes de Fratura do Rádio Distal. Clínica Ortopédica e Pesquisas Relacionadas, v. 475, n. 3, p. 595-596, 2017.

19. BRASIL. Ministério da Saúde. Sistema Nacional de Auditoria (SNA), Auditoria do SUS no contexto da SNA. Qualificação do relatório de auditoria. Brasília, 2015.

20. BRASIL. Ministério da Saúde. Portaria № 2.567, de 25 de Novembro de 2016: dispõe sobre a participação complementar da iniciativa privada na execução de ações e serviços de saúde e o credenciamento de prestadores de serviços de saúde no Sistema Único de Saúde (SUS). Disponível em: http://bvsms.saude.gov.br/bvs/saudelegis/gm/2016/prt2567 2511 2016.ht $\underline{\mathrm{ml}}$

21. FONTES, Thaís Alves; SELOW, Marcela Lima Cardoso. Ética no Serviço Público e Controle Interno. Vitrine de Produção Acadêmica Produção de Alunos da Faculdade Dom Bosco, v. 4, n. 2, 2017.

22. RODRIGUES, João Gaspar. Publicidade, transparência e abertura na administração pública. Revista de Direito Administrativo, v. 266, p. 89123, 2014.

23. CBHPM-2016: Classificação Brasileira Hierarquizada de Procedimentos Médicos / Associação Médica Brasileira. - Barueri, SP: Manole; São Paulo: Associação Médica Brasileira, 2016.

24. GUERRER, Gabriela Favaro Faria; CASTILHO, Valéria; LIMA, Antônio Fernandes Costa. Processo de formação de contas em um hospital de ensino especializado em cardiologia e pneumonia. Revista Eletrônica de Enfermagem, v. 16, n. 3, p. 558-65, 2014. 
25. COFEN. Conselho Federal de Enfermagem. Resolução № 429/2012:

dispõe sobre o registro das ações profissionais no prontuário do paciente, e em outros documentos próprios da enfermagem, independente do meio de suporte - tradicional ou eletrônico. Disponível em:

http://www.cofen.gov.br/resoluo-cofen-n-4292012 9263.html.

Recebido: 27 de novembro de 2018. Publicado: 28 de dezembro de 2018

Correspondência: Eduardo Neves da Cruz de Souza. E-mail:

educruzz@live.com

Conflito de Interesses: os autores declararam não haver conflito de interesses.

(C) This is an Open Access article distributed under the terms of the Creative Commons Attribution License, which permits unrestricted use, distribution, and reproduction in any medium, provided the original work is properly cited 